\title{
Estenosis congénita aislada del conducto auditivo interno como causa de hipoacusia neurosensorial unilateral y paresia del nervio facial

\author{
Congenital internal auditory canal stenosis presenting with unilateral \\ sensorineural hearing loss and facial nerve dysfunction
}

\author{
Sofía Waissbluth A ${ }^{1}$, Sebastián Bravo G² , Catalina Gutiérrez C³ .
}

\begin{abstract}
RESUMEN
La estenosis del conducto auditivo interno con aplasia/hipoplasia del nervio cocleovestibular es una patología muy infrecuente. Suele ser unilateral y puede acompañarse de aplasia/hipoplasia del nervio facial y otras malformaciones del oído interno. Se presentan aquí dos casos clínicos de pacientes pediátricos con estenosis del conducto auditivo interno unilateral con compromiso del séptimo y octavo par craneal ipsilateral. Se describen las historias y evaluaciones clínicas, hallazgos audiovestibulares, hallazgos imagenológicos, tratamientos indicados y sus resultados.

Palabras clave: Estenosis del conducto auditivo interno, hipoplasia del nervio cocleovestibular, parálisis facial, hipoacusia.
\end{abstract}

\begin{abstract}
Congenital internal auditory canal stenosis associated with aplasia/hypoplasia of the cochleovestibular nerve is a very infrequent pathology. It is usually unilateral and may be accompanied by aplasia/hypoplasia of the facial nerve and other malformations of the inner ear. We hereby present two clinical cases of pediatric patients with congenital internal auditory canal stenosis, with involvement of the seventh and eighth ipsilateral cranial nerve. The medical histories and clinical evaluations, audiovestibular findings, imaging findings, treatments and their results are described.
\end{abstract}

Key words: Congenital internal auditory canal stenosis, hypoplasia of the cochleovestibular nerve, facial nerve paralysis, hearing loss.

\footnotetext{
Departamento de Otorrinolaringología, Pontificia Universidad Católica de Chile, Santiago, Chile.

Departamento de Neurorradiología, Pontificia Universidad Católica de Chile, Santiago, Chile.

3 Departamento de Otorrinolaringología, Complejo Asistencial Dr. Sótero del Rio, Santiago, Chile.

Los autores declaran no tener conflictos de interés.
}

Recibido el 10 de mayo, 2019. Aceptado el 29 de mayo, 2019. 


\section{INTRODUCCIÓN}

La estenosis congénita del conducto auditivo interno es una patología infrecuente. Se presenta generalmente como hipoacusia neurosensorial unilateral, aunque puede acompañarse de alteraciones de otros pares craneales ${ }^{1}$. El uso de imágenes contribuye al diagnóstico y son indispensables para descartar otros diagnósticos diferenciales de hipoacusia neurosensorial unilateral congénita ${ }^{2,3}$. Aquí presentamos dos casos clínicos de estenosis congénita del conducto auditivo interno con hipoacusia sensorioneural unilateral significativa acompañada de una paresia del nervio facial ipsilateral. Describimos la historia clínica, los hallazgos audiovestibulares e imagenológicos que apoyaron el diagnóstico y el tratamiento indicado.

\section{CASO CLÍNICO 1}

Paciente de sexo masculino de 10 años, con antecedentes de síndrome de alcoholismo fetal, fue derivado a nuestro departamento de otorrinolaringología para evaluación de la audición. Según la madre del paciente, se sometió a una prueba de potenciales evocados auditivos de tronco cerebral (PEATC) que resultó anormal para el oído izquierdo, siete años antes. El examen físico otorrinolaringológico mostró una hipofunción del nervio facial del lado izquierdo, grado IV de House-Brackmann. La impedanciometría mostró timpanogramas tipo A bilaterales con ausencia del reflejo acústico para el oído izquierdo, y la presencia del reflejo para el oído derecho (los reflejos medidos ipsilateralmente). En la audiometría, se observó una pérdida completa de audición en el oído izquierdo con $0 \%$ de discriminación del habla, mientras que el promedio de tonos puros (PTP) para el oído derecho fue de $11,3 \mathrm{~dB}$ con $100 \%$ de discriminación del habla (Figura 1a).

Las emisiones otoacústicas por productos de distorsión no se detectaron en el oído izquierdo. Además, al realizar una electrococleografía evocada por estímulo click, no se observó actividad para el oído izquierdo (electrodo colocado en el conducto auditivo externo). Se realizó una prueba de impulso cefálico asistida por video (vHIT) para los canales semicirculares horizontales; la ganancia del reflejo vestíbulo-ocular (RVO) estuvo dentro del rango normal para el lado derecho, y disminuido para el lado izquierdo $(0,36)$. Sacadas correctivas (cubiertas y descubiertas) también fueron detectadas para el lado izquierdo (Figura 2a).

La tomografía computarizada (TC) del hueso temporal de alta resolución reveló un conducto auditivo interno estenótico en el lado izquierdo (menos de 2 $\mathrm{mm})$, sin otras anomalías asociadas del oído interno. Los segmentos cisternales del nervio cocleovestibular y facial no se pudieron observar en la resonancia magnética (RM) ponderada en T2 (Figura 3).

Se decidió adaptar al paciente con un audífono tipo CROS (presentación contralateral de la señal). Al principio, el paciente tenía dificultades para adaptarse al audífono, pero después de un par de meses de uso, dio buenos resultados.

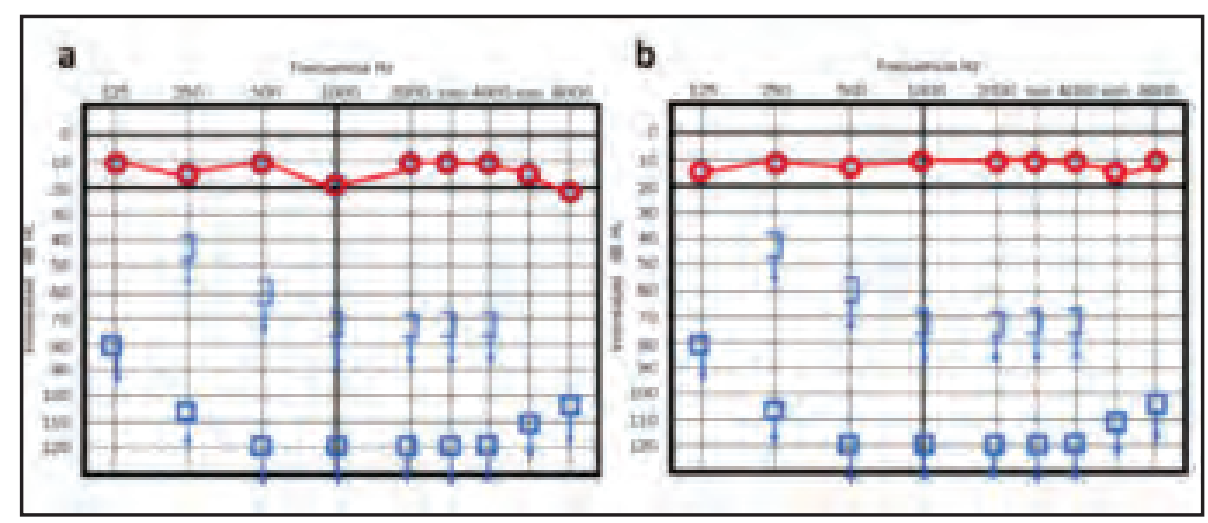

Figura 1. Audiometría tonal. (a) Paciente \#1; se observa cofosis del oído izquierdo y promedio de tonos puros para el oído derecho de 11,3 dB. (b) Paciente \#2; se observa una situación similar al paciente \#1, con cofosis del oído izquierdo y un promedio de tonos puros de $11,3 \mathrm{~dB}$ para el oído derecho. 


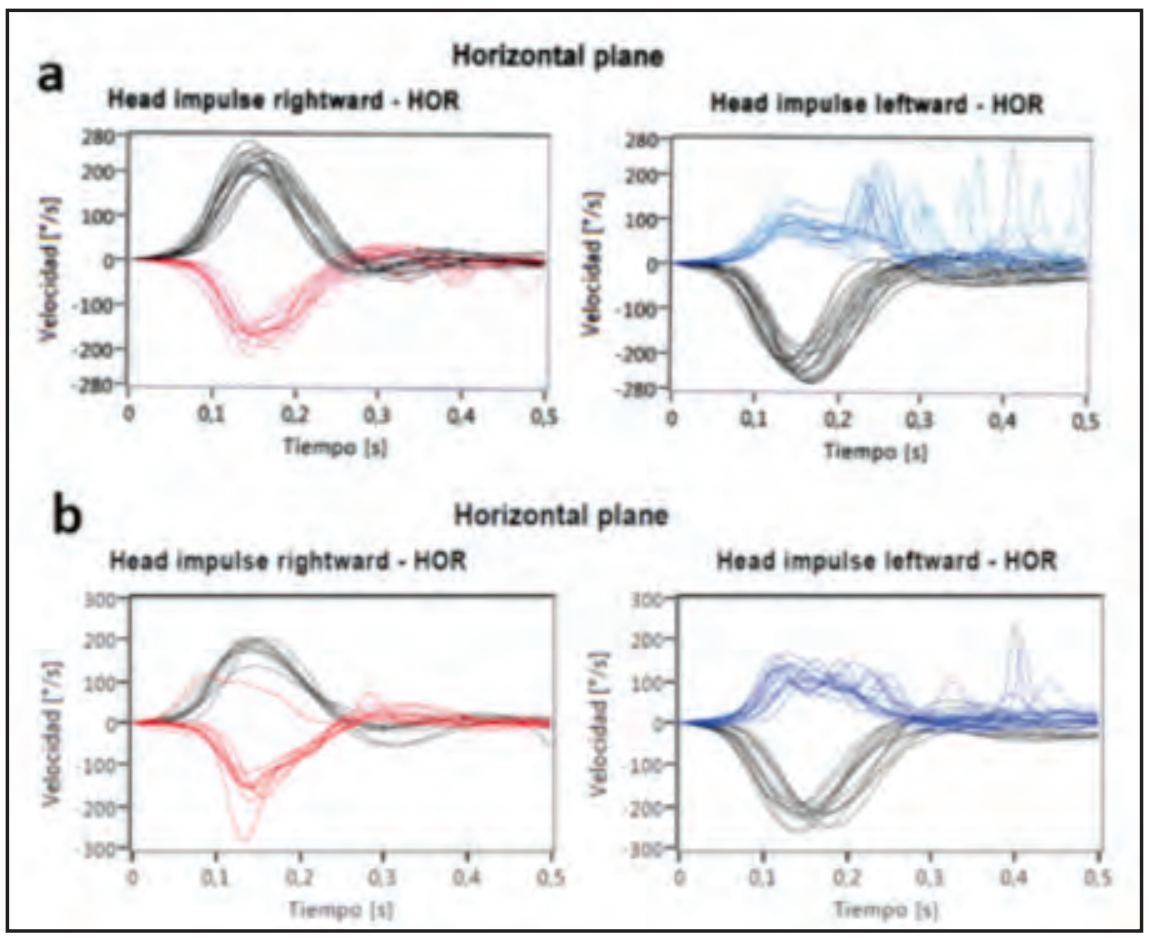

Figura 2. Prueba de impulso cefálico asistida por video para los canales semicirculares horizontales. (a) Paciente \#1; el RV0 está dentro del rango normal para el lado derecho $(0,71)$, y disminuido para el lado izquierdo $(0,36)$. (b) Paciente \#2; RV0 está dentro del rango normal para el lado derecho $(0,70)$, y disminuido para el lado izquierdo $(0,60)$. Se observan sacadas cubiertas y descubiertas para el canal semicircular horizontal izquierdo de ambos pacientes.

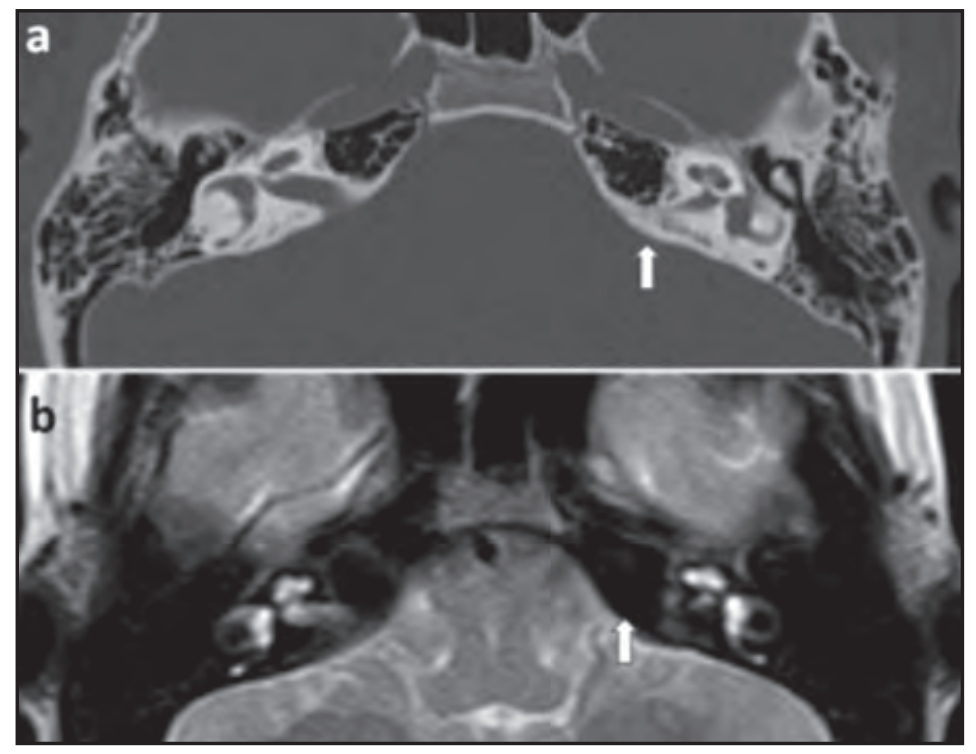

Figura 3. Paciente \# 1. (a) TC, corte axial. La flecha blanca indica el conducto auditivo interno estenótico en el lado izquierdo. No se observan otras malformaciones del oído interno. (b) RM potenciada en T2, corte axial. La flecha blanca indica el área del conducto auditivo interno estenótico; el nervio cocleovestibular no se logra distinguir. 


\section{CASO CLÍNICO 2}

Paciente de sexo femenino de 8 años de edad fue atendida en el departamento de otorrinolaringología con una historia de emisiones otoacústicas alteradas en el período neonatal para el oído izquierdo, también presentó una disfunción del nervio facial del lado izquierdo, House-Brackmann grado IV. A los 8 meses de vida, se realizó una RM que mostró hipoplasia del conducto auditivo interno izquierdo y un canal semicircular lateral dilatado en el lado izquierdo. No se pudo observar el nervio facial y ni coclear ipsilateral (Figura 4).

Una TC de hueso temporal de alta resolución reveló un conducto auditivo interno estenótico en el lado izquierdo. La impedanciometría mostró timpanogramas tipo A bilaterales con ausencia del reflejo acústico para el oído izquierdo, pero presente para el oído derecho (los reflejos se miden ipsilateralmente). En la audiometría, se observó una pérdida completa de la audición con $0 \%$ de discriminación del habla para el oído izquierdo, mientras que el PTP para el oído derecho fue de $11,3 \mathrm{~dB}$ con $100 \%$ de discriminación del habla (Figura 1b). Una prueba de vHIT para los canales semicirculares horizontales mostró una ganancia de ROV dentro del rango normal para el lado derecho, y disminuido para el lado izquierdo $(0,59)$ con sacadas cubiertas y descubiertas (Figura $2 \mathrm{~b}$ ).
Al hacer una electrococleografía con estímulo click (electrodo colocado en el conducto auditivo externo), se observaron formas de onda anormales y una alta relación SP:AP [SP: potencial de sumación, AP: potencial de acción] para el oído izquierdo. Se adaptó con un audífono tipo CROS que proporcionó resultados adecuados.

\section{DISCUSIÓN}

Presentamos dos casos clínicos de estenosis congénita del conducto auditivo interno con pérdida auditiva neurosensorial unilateral y paresia del nervio facial ipsilateral. Uno de los pacientes también presentó una malformación del oído interno asociada: un canal semicircular lateral dilatado. Ambos pacientes presentaron cofosis unilateral con una disminución de la ganancia del RVO ipsilateral. La obtención de imágenes con RM y TC reveló la etiología, un conducto auditivo interno estenótico unilateral.

La estenosis congénita del conducto auditivo interno es rara, y puede verse junto con otras anomalías del oído interno. Por lo general, es una afección unilateral que se presenta con una pérdida auditiva neurosensorial profunda'. Tanto la RM como la TC de hueso temporal son necesarias para realizar un diagnóstico adecuado, ya que la

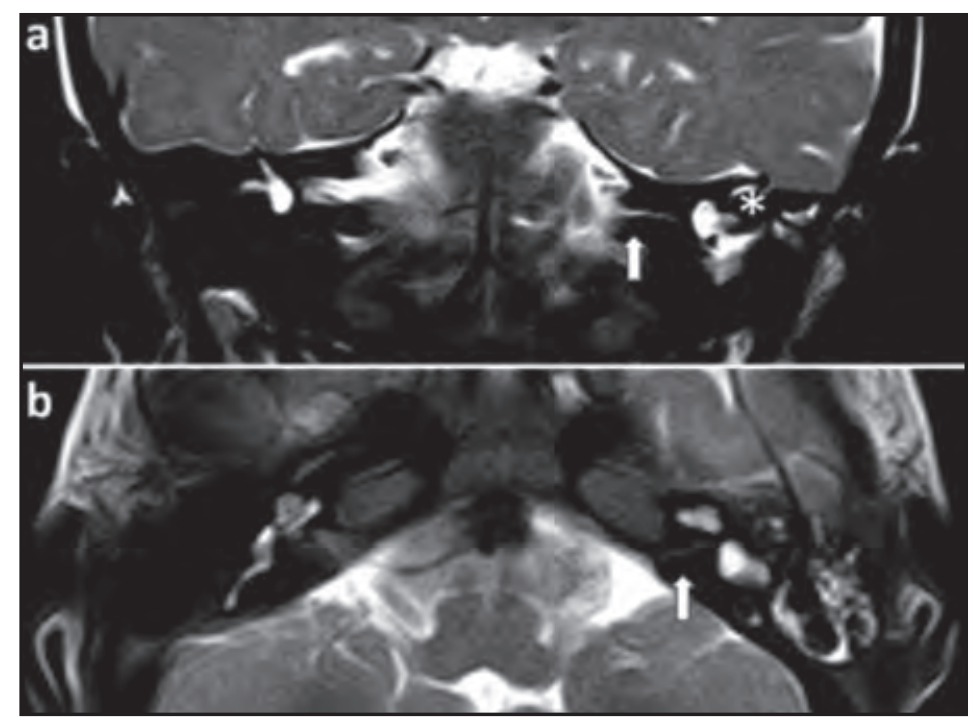

Figura 4. Paciente \# 2. (a) RM potenciada en T2, corte coronal, y (b) corte axial. La flecha blanca indica hipoplasia del conducto auditivo interno izquierdo. El * indica el canal semicircular lateral dilatado en el lado izquierdo. 
TC de alta resolución ve detalles óseos complejos y malformaciones del oído interno, mientras que la RM de alta resolución ponderada en T2 permite la visualización del laberinto membranoso, el nervio cocleovestibular, y también ayuda a excluir causas centrales de la pérdida auditiva neurosensorial congénita ${ }^{2,3}$. Las imágenes también son cruciales para determinar el manejo, ya que la ausencia del nervio coclear es una contraindicación para el implante coclear $^{2,4}$, por lo que los hallazgos altamente sugerentes en las imágenes junto con las pruebas de audición funcionales anormales son de utilidad para determinar el tratamiento. Se cree que un conducto estenótico es secundario a la falta de desarrollo del nervio cocleovestibular, y un diámetro de menos de $2 \mathrm{~mm}$ se considera estenótico (rango normal 2-8 mm ${ }^{3}$. Uno de los pacientes también se presentó con una dilatación del canal semicircular lateral, éste es el último canal en formarse. Se ha sugerido que una malformación de un canal semicircular con una cóclea normal es posiblemente el resultado de una alteración que ocurre entre la séptima y la $22^{a}$ semana de gestación ${ }^{2}$. Aunque el nervio facial viaja por dentro del conducto auditivo interno, su desarrollo es independiente del nervio cocleovestibular $^{5}$, y la disfunción del nervio facial

\section{BIBLIOGRAFÍA}

1. Yates Ja, Patel PC, Millman B, Gibson WS. Isolated congenital internal auditory canal atresia with normal facial nerve function. Int J Ped Otol 1997; 41: 1-8.

2. Casselman JW, Offeciers EF, De Foer B, Govaerts P, Kuhweide R, Somers T. CT and MR imaging of congenital abnormalities of the inner ear and internal auditory canal. Eur J Radiol 2001; 40: 94-104.

3. Joshi VM, Navlekar SK, Kishore GR, Reddy KJ, KumAR EC. CT and MR Imaging of the Inner Ear and Brain in Children with Congenital Sensorineural Hearing Loss. RadioGraphics 2012; 32: 683-98.

4. Manrique M, Ramos Á, de Paula Vernetta C, ET AL. Guideline on Cochlear Implants. Acta Otorrinolaringol Esp 2019; 70: 47-54. es poco común en la estenosis congénita del conducto auditivo interno ${ }^{6}$. Ambos casos descritos aquí presentan paresia del nervio facial ipsilateral.

La estenosis congénita del conducto auditivo interno es una causa poco frecuente de pérdida auditiva neurosensorial. Los pacientes también pueden presentar síntomas vestibulares y/o disfunción del nervio facial. Las imágenes con TC y RM de alta resolución son esenciales para el diagnóstico y también para determinar una estrategia de manejo. La pérdida auditiva profunda unilateral en pacientes pediátricos se debe tratar, ya que puede afectar el desarrollo del habla y del lenguaje. Estos pacientes, sus familias y sus profesores deben ser educados sobre el tema. Se debe igualmente ofrecer un dispositivo auditivo, como por ejemplo un audífono con presentación contralateral de la señal (ejemplo CROS) 0 un procesador de sonido de conducción ósea ${ }^{7}$. Dependiendo de la disponibilidad, se puede considerar realizar un PEATC con estímulo eléctrico (grabación de ventana redonda o promontorio) para niños pequeños cuando no se logra una respuesta luego de un PEATC obtenido mediante estimulación acústica convencional, debido a que la implantación coclear se ha intentado en niños con aplasia o hipoplasia del nervio coclear, $\mathrm{y}$ ha dado algunos resultados prometedores ${ }^{8}$.

5. Glastonbury CM, Davidson hC, Harnsberger HR, Butler J, Kertesz TR, Shelton C. Imaging Findings of Cochlear Nerve Deficiency. AJNR Am J Neuroradiol 2002; 23: 635-43.

6. Baek SK, Chae SW, Jung HH. Congenital internal auditory canal stenosis. J Laryngol Otol 2003; 117: 784-7.

7. Purcell PL, Jones-Goodrich R, Wisneski M, Edwards TC, SiE KC. Hearing devices for children with unilateral hearing loss: Patientand parent-reported perspectives. Int J Pediatr Otorhinolaryngol 2016; 90: 43-4.

8. Ehrmann-Müller D, Kühn H, Matthies C, Hagen R, Shehata-Dieler W. Outcomes after cochlear implant provision in children with cochlear nerve hypoplasia or aplasia. Int J Pediatr Otorhinolaryngol 2018; 112: 132-40.

\author{
Correspondencia: Catalina Gutiérrez Cisternas \\ Departamento de Otorrinolaringología, Complejo Asistencial Dr. Sótero del Río \\ Av. Concha y Toro 3459, Puente Alto, Santiago, Chile \\ E mail: catagutierrezci@gmail.com
}

